\title{
Heparan sulfate proteoglycans: heavy hitters in the angiogenesis arena
}

\author{
Renato V. Iozzo ${ }^{1,2}$ and James D. San Antonio ${ }^{3}$ \\ ${ }^{1}$ Department of Pathology, Anatomy and Cell Biology, \\ ${ }^{2}$ Cellular Biology and Signaling Program, Kimmel Cancer Center, and \\ ${ }^{3}$ Department of Medicine, Cardeza Foundation, Thomas Jefferson University, Philadelphia, Pennsylvania, USA \\ Address correspondence to: Renato V. Iozzo, Department of Pathology, Anatomy and Cell Biology, Room 249, Jefferson Alumni Hall, \\ Thomas Jefferson University, 1020 Locust Street, Philadelphia, Pennsylvania 19107, USA. \\ Phone: (215) 503-2208; Fax: (215) 923-7969; E-mail: iozzo@lac.jci.tju.edu.
}

J. Clin. Invest. 108:349-355. DOI:10.1172/JCI200113738.

Without new blood vessels, neoplasms cannot expand beyond a few millimeters, the point at which the diffusion of nutrients and the disposal of waste products become rate-limiting. Regulation of angiogenesis thus must be controlled at multiple levels. For instance, the VEGF family of heparin-binding proteins and their primary receptors, VEGFR-1 and VEGFR-2 (KDR), products of the $f l t-1$ and the $f l k-1$ gene, respectively, are required for angioblast differentiation and vasculogenesis, and specific VEGF isoforms play distinct roles in promoting endothelial growth and migration during angiogenesis. In addition, angiogenesis is profoundly affected by several members of the FGF family and their four receptors, and indeed, supplementing the media of endothelial cell cultures with basic FGF (FGF2) and heparin is now well established as a means to obtain optimal growth, migration, and capillary morphogenesis. In addition to producing proangiogenic factors, tumor cells also directly or indirectly generate negative angiogenic stimuli. The ultimate growth rate of the tumors is thus a fine balance between positive and negative angiogenic cues.

Heparan sulfate proteoglycans (HSPGs) act in concert with members of the FGF and VEGF families and their receptors to control various aspects of vascular development and tumor angiogenesis. A processed form of the newly discovered HSPG-collagen hybrid molecule collagen type XVIII can exert strong antitumor activity by reversibly blocking tumor angiogenesis. This Perspective will focus primarily on the roles of this and other HSPGs on angiogenesis, with special emphasis on the activity of the protein core, its functional partners, and its processed isoforms.

The players: heterogeneity is the norm

The enormous heterogeneity of HSPGs makes these macromolecules some of the most complex substances of structured tissues (1-4). These adaptable molecules interact with cells or other binding partners via their heparan sulfate chains, protein cores, or both. There is ample evidence that endogenous and tumor-induced neovascularization is quite heteroge- neous. In the former case, for example, it has long been appreciated that some patients are good while others are poor collateral formers, as seen in the formation of new vessels in cardiac tissues near sites of myocardial infarcts. This variable response may be due, at least in part, to individual variations in the structure of certain key HSPG players (Figure 1).

Cell surfaces and the immediate neighboring structures, such as basement membranes, contain several

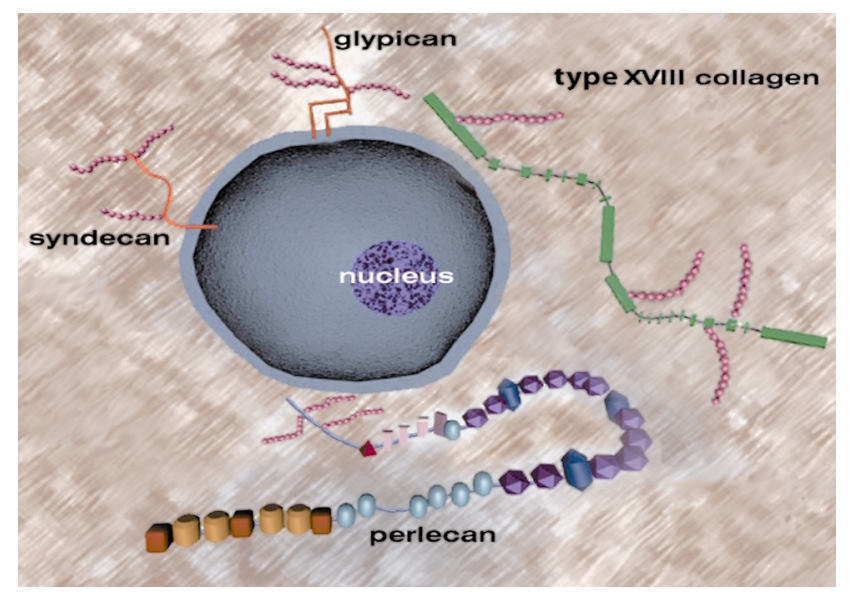

\section{Figure 1}

Heterogeneity in structure of pericellular and cell-associated heparan sulfate proteoglycans with pro- and antiangiogenic activity. Syndecans are transmembrane proteoglycans, while glypicans are bound to the plasma membrane via a phosphoinositol linkage. Type XVIII collagen, a hybrid collagen/proteoglycan molecule, is a member of the multiplexin gene family characterized by alternating collagenous (thin lines) and noncollagenous domains (rectangles, not drawn in scale). Perlecan is a modular proteoglycan with five distinct domains. Both type XVIII collagen and perlecan are secreted products but are in close association with the cell surface and are intrinsic components of most basement membranes. Agrin, another HSPG with similarities to perlecan, is not shown. Glycosaminoglycan chains are shown as fuchsia-colored beaded strings and are not drawn to scale relative to their respective protein cores. The precise location and number of the heparan sulfate chains on type XVIII collagen are not known. 
classes of HSPGs. Syndecans 1-4 are transmembrane proteins that carry heparan sulfate chains near their extracellular tips and sometimes carry chondroitin sulfate chains near the cell surface (3). Glypicans 1-6 are attached to the plasma membrane via a glycosyl phosphoinositol linkage and may carry several heparan sulfate side chains near the plasma membrane, and sometimes an additional chain is attached near the end of its ectodomain (5).

There are at least three additional classes of pericellular HSPGs. Agrin, an abundant HSPG of most basement membranes, is also located in the synaptic region and functions in neurotransmission. Its role in angiogenesis has not been investigated in depth, but given its strategic location and ubiquitous nature, we can predict that it might also play a role in modulating activities of heparin-binding angiogenic proteins. Intriguingly, agrin can also occur as an intercalated HSPG in the CNS when an alternate transmembrane domain is spliced into its $\mathrm{N}$-terminus, thereby converting a basement membrane HSPG into a type II transmembrane HSPG (6). Whether a similar mechanism applies to other basement membrane molecules is not known, but there is sequence evidence for three nidogen isoforms with potential transmembrane regions in their C-terminal domains. Another class of pericellular HSPGs includes perlecan, a modular proteoglycan with a widespread tissue distribution and a very complex structure (4). The modules of perlecan have a significant homology to proteins involved in lipid uptake, growth-promoting activity, homotypic and heterotypic interactions, and adhesion. Perlecan usually carries three heparan sulfate chains near the $\mathrm{N}$-terminus, but it could also carry chondroitin sulfate chains near the C-terminus and exhibit a complex pattern of alternative splicing in Caenorbabditis elegans (7). The third class encompasses collagen type XVIII, a hybrid collagenHSPG molecule that is a constituent of most basement membranes and is particularly abundant in kidneys and the peripheral nervous system. Collagen XVIII belongs to the multiplexin gene family characterized by multiple collagenous domains (triple-helical regions) interrupted and flanked by nontriple-helical domains (8). This proteoglycan may carry up to four heparan sulfate chains, each attached to one of the 11 noncollagenous domains (Figure 1). The recent discovery that collagen type $\mathrm{XV}$, the second member of the multiplexin gene family, is also a hybrid proteoglycan carrying chondroitin sulfate chains (9) indicates that the noncollagenous domains of these molecules are highly suitable for glycosaminoglycan substitution.

Thus, a characteristic feature of pericellular HSPGs is their structural heterogeneity. This complexity in structure is further increased by generation of splice variants, proteolytically processed and differentially glycosylated isoforms.

\section{Durable signaling}

Two salient features of pericellular HSPGs are their longevity and high concentration. These macromolecules can have half-lives of hours to days and can often reach micromolar concentrations, in contrast to growth factors and cytokines which are often short-lived and occur at nanomolar concentrations. Even in the case of shedding of cell surface HSPGs, the entire ectodomain is often released with important biological effects (3). Consequently, the HSPG-mediated signaling events are often sustained, function as autocrine regulators of growth, and involve a paracrine action. Moreover, pericellular HSPGs can filter developmental cues, such as Wingless $(\mathrm{Wg} / \mathrm{Wnt}$ ) growth factors, by forming charged gradients to which growth factors bind with variable affinities (10). The amount of a distinct HSPG needs to be precisely monitored to ensure proper patterning, inasmuch as HSPG concentrations can affect morphogen distribution and thereby function. Consistent with this model, a dramatic decrease of extracellular Wingless is also detected in clones of cells that are deficient in heparan sulfate biosynthesis (11).

The signaling pathways activated by FGFs and VEGFs are essentially heparan sulfate-dependent, as evidenced by the failure of cells deficient in heparan sulfate biosynthesis to activate these pathways and the ability of exogenous heparin/HSPG to restore their function. The primary defect in the heparan sulfate-deficient cells occurs at the initiation of the signaling cascade and is likely caused by an abnormal receptor occupancy or a failure to induce a proper association or conformation of the receptor-ligand complexes. Because heparan sulfate is the most abundant glycosaminoglycan form, whereas heparin is confined to mast cells, and because heparan sulfates are highly versatile and heterogeneous structures compared with heparin, it is likely that the in vivo signaling events are mediated by heparan sulfate and that these biological interactions are even more complex than previously suspected. The recent development of novel analytical approaches to decode heparan sulfate sequence will undoubtedly advance our knowledge of key interactions.

A common requirement for all tyrosine kinase receptors is the formation of a threshold number of phosphorylated cytoplasmic domains to initiate a specific signaling cascade. This implies that a threshold number of active receptor-ligand complexes also needs to be present on the cell surface for a suitable period of time to result in effective signaling. Whether the key HSPG function in signaling is due to a sustained coactivation or to a simple limitation of extracellular diffusion of growth factors and morphogens, the take-home message is the same: HSPGs transduce signals in a way that differs dramatically from the known action of hormones and cytokines. This raises the transduction of biological signals to an even higher level of complexity.

\section{Potentiating and inhibiting activities}

An emerging key concept is that HSPGs can act as activators or suppressors of FGF activities. Physiologically, cells can induce or suppress HSPG expression, which in turn modulates the nature and strength of signaling from the various FGFs stored in the matrix. For instance, perlecan induces high-affinity binding of FGF2 to heparan sulfate-deficient cells and augments FGF2 mitogenic activity (12), while glypican-1 inhibits the binding of FGF7 (keratinocyte growth fac- 
tor) to its receptor (13). Activated macrophages overexpress syndecan-2, which selectively binds FGF2 and VEGF and presents FGF2 in a form that transactivates receptor-bearing cells (14). In inflammatory macrophages the HSPG form of CD44, the major cellsurface receptor for hyaluronan, functions as a paracrine regulator of FGF action: it binds the macrophage-derived FGF2 and VEGF and, in the former case, stimulates productive binding to the highaffinity FGF receptor 1 (15). Notably, CD44 HSPG and syndecan- 2 are abnormally expressed in the synovial membranes and subintimal macrophages, thus suggesting that these HSPGs may be directly involved in the pathogenesis of inflammatory arthritis.

Low concentrations of heparin/heparan sulfate stimulate FGF7-mediated signaling, while higher concentrations inhibit FGF7- but do not affect FGFR1-mediated signaling. These biphasic effects suggest that occupancy of the HSPG binding sites on the receptor may specifically inhibit FGF7 action. The ability of heparan sulfate to alternatively stimulate or inhibit FGF receptor activity is likely based on a combination of the fine chemical structure of the carbohydrate chain and the physical location of the HSPG, relative to the cell surface (16). It has been proposed that the dual function of heparan sulfate in FGF2 binding can be accurately represented by a stimulation, when heparan sulfate is bound to the cell surface, and an inhibition of FGF2 binding when heparan sulfate is in solution (17). Adequate cellular responses can be achieved by appropriately adjusting the concentrations of FGF and HSPGs (18). As with other equilibrium reactions, this thermodynamic model suggests that a deficit in either component can be compensated for by increasing the effective concentration of the other (18).

A possible mechanism for inhibition could involve the sequestration of growth factors, a sort of biological quarantine where FGFs are fully active but stored in a dormant state bound to an inactivating sequence. Pericellular HSPGs could potentiate activity by physically associating with FGFs or VEGFs and their respective receptors, or they could function as chaperones, as in the case of glypican-1, which can restore the receptorbinding ability of oxidized $\mathrm{VEGF}_{165}$, a mitogen for endothelial cells and powerful angiogenic factor in vivo (19). The ultimate cellular response (stimulation or inhibition) will also depend on the concentration and binding kinetics of the growth factor, the expression of a specific HSPG and its glycosylation status, and the expression of a specific receptor and its isoforms. We believe that the functional roles that the various HSPG protein cores play in physiology and diseased states has been somewhat overlooked, in part because of the difficulty in expressing full-length products. For example, in the case of perlecan, a $467-\mathrm{kDa}$ polypeptide, producing full-length recombinant proteins presents a major technical challenge, so most investigators have expressed domain-specific portions of the parent protein core. Moreover, specific protein modules within HSPGs have high-affinity binding sites for various growth factors involved in angiogenesis (see below). Therefore, we feel that a systematic study of the HSPG protein cores should be performed to identify additional binding partners involved in the regulation of FGF and VEGF signaling pathways.

\section{HSPGs as depots for pro- and}

\section{antiangiogenic factors}

Significant roles for pericellular HSPGs in tumor-associated angiogenesis have been proposed, based in part on correlative evidence showing altered expression in the tumor stroma and the newly formed blood vessels (20). The activity of FGF2 on cell growth and vascular morphogenesis is potentiated through its association with perlecan (12). Thus, perlecan extracted from human lung fibroblasts facilitates high-affinity FGF2 binding to soluble FGF receptors, as well as to mutant, heparan sulfate-deficient Chinese ovary cells engineered to express the FGF receptor 1. Perlecan encapsulated in alginate beads binds FGF2 and promotes extensive angiogenesis in the rabbit ear chamber model. At the same time it was shown that in tumor xenografts induced by PC 3 human prostate carcinoma cells, human perlecan is deposited along the basement membrane of newly formed tumor vessels which are of obvious murine origin (20). These two observations have led to the proposals that perlecan functions as a low-affinity coreceptor by delivering FGF2 to its highaffinity receptor, and that perlecan serves as a structural scaffold for tumor angiogenesis. These activities are not mutually exclusive but complementary, since there is an ample amount of perlecan at the surface of endothelial cells linked via $\beta$ integrins and, during murine development, perlecan expression is prominent in tissues undergoing vasculogenesis (21). Consistent with this model, suppression of perlecan expression blocks autocrine and paracrine activities of FGF2 in human melanoma cells (22) and halts melanoma cell proliferation and invasion (23). These profound effects are not surprising, given the proposed roles for perlecan in lipoprotein uptake, adhesion, and thrombogenesis, all of which can influence endothelial cell migration and proliferation (4) .

These interactions are generally assumed to be mediated by the heparan sulfate chains, as in the case of the syndecans and glypicans (24). But does the enormous protein core of perlecan also play a role? Do the five modular units only function as carriers for heparan sulfate, or do they also interact with angiogenic growth factors? And if so, do other members of the FGF family interact with perlecan's protein core? To address these questions, we have generated cells with targeted suppression of perlecan gene expression using both constitutive and inducible promoters (25). Notably, attenuated perlecan expression correlates with a reduced tumor growth and angiogenesis, and this is due, at least in part, to a reduced activity of FGF7, an angiogenic growth factor that is secreted by mesenchymal cells and acts on epithelial cells. Exogenous perlecan effectively reconstitutes the activity of FGF7 in the perlecan-deficient cells. Interestingly, FGF7 binds specifically $\left(K_{\mathrm{d}} \sim 60 \mathrm{nM}\right)$ to domains III and V of human perlecan (26). Using cellfree binding experiments, a high-affinity $\left(K_{\mathrm{d}} \sim 8 \mathrm{nM}\right)$ binding site for PDGFs AA and BB has also been dis- 
covered in mouse domain III, with lower-affinity $\left(K_{\mathrm{d}}\right.$ $\sim 34-64 \mathrm{nM}$ ) sites for domains I, IV, and V (27). Thus, in spite of the internal repetitive sequences in the perlecan protein core, there are unique binding specificities for the various modules. The recent discovery that perlecan's domain III binds to FGF-BP (28), an FGF-binding protein that modulates the activity of FGF2 and FGF7 and acts as an angiogenic switch $(29,30)$, further strengthens this concept. Clearly, not only members of the FGF family but also proteins that modulate FGF activity bind domain-specific perlecan sequences.

The dual theme of stimulation and inhibition recurs elsewhere, as demonstrated by two independent studies. In HT1080 fibrosarcoma cells, suppression of perlecan expression results in stimulation of tumor cell growth, both in vitro and in vivo, increased invasion of ECM, and enhanced adhesion to type IV collagen substrata (31). These effects are independent of FGF 2 and suggest that perlecan exerts negative regulatory functions in mesenchymal-derived tumors such as fibrosarcomas. In an immortalized cell line derived from Kaposi's sarcoma, suppression of perlecan expression causes enhanced cell migration and proliferation in vitro and enhanced tumorigenesis and angiogenesis in tumor xenografts (32). A plausible explanation for these results is that lack of perlecan in these two sarcomas favors the diffusion of heparin-binding growth and angiogenic factors, thereby promoting tumorigenesis. The cellular context, thus, plays a major role in perlecan's functions.

Perlecan itself can be classified as a heparin-binding protein, and indeed it binds heparin and sulfatides via sequences that have been mapped to domain V. Moreover, it binds a variety of growth factors and cytokines including HGF, ILs, platelet factor 4, TGF- $\beta$, and IFN- $\gamma$ (4). Perlecan gene expression can also be regulated by these factors. IFN- $\gamma$, for example, rapidly and efficiently silences perlecan transcription via Stat1-binding sites in the distal promoter region (33). Thus, the IFN- $\gamma$-mediated transcriptional repression of perlecan is an additional antitumor effect of this cytokine, through which it eliminates a powerful angiogenic stimulus from the tumor microenvironment.

Proteoglycan-mediated FGF and VEGF potentiation may occur via distinct sites on both the heparan sulfate chains and the protein core. At the cell surface, HSPGs contribute to the formation of a ternary complex with two FGF molecules and one growth factor receptor chain, which form an "activated" unit (34). The HSPGs can also serve as important reservoirs for other angiogenic factors. For example, cell surface HSPGs have been proposed to be docking sites for matrix metalloproteinase 7 (MMP-7), which, when released, can initiate endothelial cell migration during angiogenesis (35). Indeed, FGF2 can be released from the $\mathrm{N}$-terminal heparan sulfate chains of perlecan by the concerted action of proteases (including stromelysin, collagenase, plasmin, and elastase) and heparanases (36). FGF-BP bound to domain III of human perlecan can seemingly be liberated at the sites of tumor invasion (28), thereby potentiating the activity of FGF2 and FGF7 and, thus, promoting angiogenesis. Obviously, some of these events described for perlecan could include other pericellular HSPGs.
Perhaps the most far-reaching role for HSPGs in angiogenesis is associated with the hybrid proteoglycan/collagen type XVIII. This molecule came into focus of medical attention when its 18-kDa C-terminal peptide, named endostatin to indicate its ability to block endothelial cell growth, was shown to have tumor-suppressive and antiangiogenic activity (37). Three-dimensional studies of recombinant endostatin revealed a compact globular structure with homology to C-type lectin, which can be liberated from the parent molecule through the action of proteases such as cathepsin $\mathrm{L}$ and certain MMPs (8). Endostatin inhibits FGF2-induced stimulation of endothelial cell growth and inhibits the growth and associated angiogenesis of lung carcinomas, melanomas, fibrosarcomas, and endotheliomas (37). In agreement with these studies, tumor progression correlates with a decreased expression of collagen type XVIII in hepatocellular carcinoma (38). Notably, analysis of the residual tumors in endostatin-treated mice revealed an increase in apoptosis, presumably via inhibition of tumor angiogenesis (37). Because discontinuation of endostatin treatment results in regrowth of the implanted xenografts, endostatin might represent a tumor dormancy factor. Recent therapeutic attempts using either microencapsulated endostatin-secreting cells or adenovirus vectors have been partially successful in retarding the growth of gliomas or suppressing liver and pulmonary metastases, respectively.

At present, the exact molecular mechanism and the target receptor for endostatin-mediated functions are not known. Somewhat controversial results point toward an indirect mode of action, likely mediated by a block in FGF or VEGF signaling. For example, human recombinant endostatin inhibits endothelial cell migration in response to VEGF and prevents the growth of renal cell carcinoma xenografts (39). Mutation of either the zinc-binding site, which originally was thought to be necessary for endostatin's function, or the heparinbinding regions was ineffective (39). In another study, mouse recombinant endostatin inhibited FGF2-mediated, but not VEGF-mediated, angiogenesis in a chorioallantoic membrane assay (40). Although mutation of the zinc-binding region had no effect, the mutational block of heparin-binding sites decreased endostatin activity. Endostatin may also act by disrupting integrin function during endothelial cell migration by acting as a ligand for $\alpha_{5}$ and $\alpha_{V}$ integrins (41). When immobilized on a solid substratum, endostatin promotes adhesion, motility, and survival. In contrast, soluble endostatin acts as an integrin antagonist and inhibits these processes (41). Another proposed mode of action of endostatin is through a blockage of the activation of MMP-2, which in turn would inhibit the breaching of basement membranes and thus prevent proper angiogenesis and tumor development (42). Notably, the endostatin analogue from collagen XV, another member of the multiplexin gene family, interacts with neither zinc nor heparin, but nonetheless inhibits FGF2- and VEGF-mediated angiogenesis (43). In addition, strikingly different activities have been reported in the ability of endostatin derived from either collagen XV or XVIII to block FGF2 and VEGF activities. 

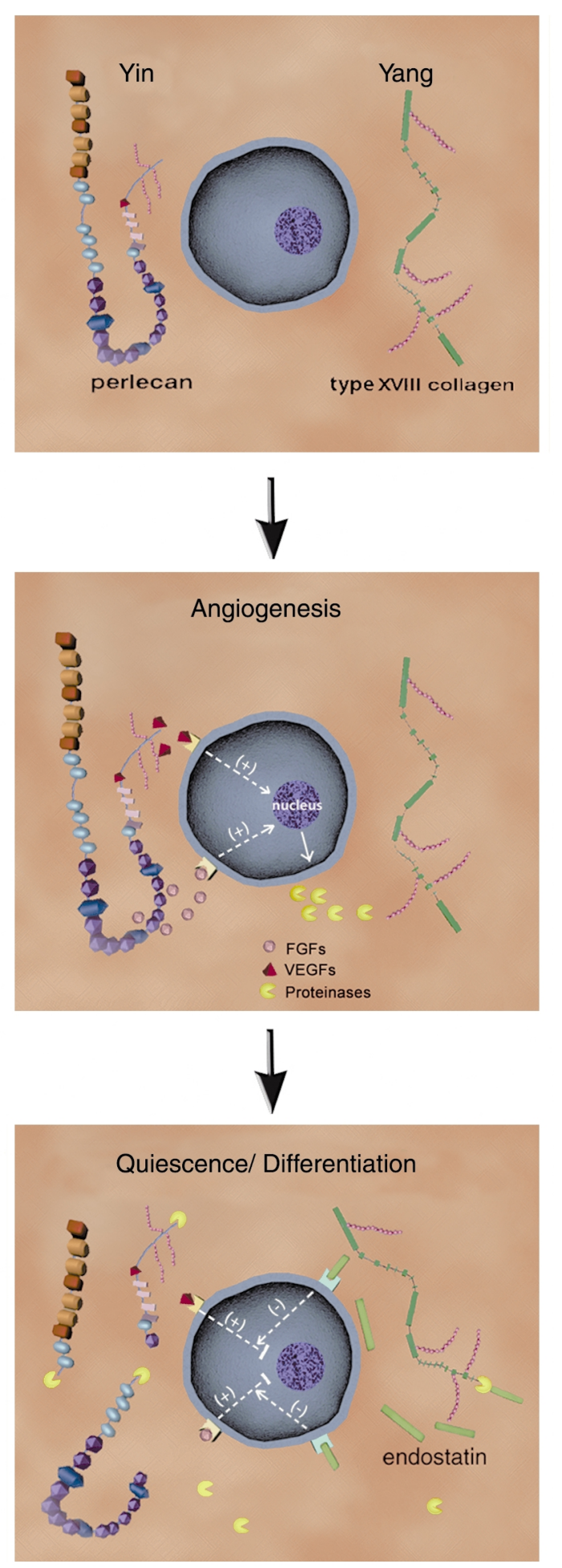

It is intriguing that endostatin binds heparin, albeit with moderate affinity $\left(K_{\mathrm{d}} \sim 300 \mathrm{nM}\right)(8)$. In vivo, however, endostatin would have ample opportunity to interact with the heparan sulfate chains of collagen XVIII itself, since the average length of the heparan sulfate chains could account for a true "overlap" with the C-terminal endostatin. Indeed, heparan sulfate chains can extend far beyond the length of relatively large protein cores. In addition, endostatin could

\section{Figure 2}

The yin (perlecan) and yang (type XVIII collagen) of angiogenic regulation by heparan sulfate proteoglycans (top panel). Perlecan, syndecans, and glypicans are proposed to act as coreceptors to deliver proangiogenic factors to their high-affinity cell surface receptors. As shown for perlecan, angiogenic stimulation might follow from its increased expression or cell surface accessibility. This would result in release of growth factors such as VEGF, carried by specific heparan sulfate sequences, or FGF2 and FGF7, bound to either heparan sulfate chains or unique regions of the protein core (middle panel). These events promote the cell proliferation phase of angiogenesis and may also trigger the synthesis and release of a battery of proteinases into the ECM milieu. Proteinases in turn would degrade perlecan, thus attenuating its growth-promoting activities, and liberate endostatin from type XVIII collagen (bottom panel). Endostatin is proposed to block VEGF- and FGF-mediated angiogenic signaling, thereby maintaining the endothelium in a quiescent state.

interact with the heparan sulfate chains of nearby proteoglycans, such as agrin and perlecan, which often codistribute in the basement membrane zones of various organs. In situ binding studies using soluble endostatin and frozen sections of human tissues have shown a specific localization to the subendothelial matrix. However, Chang et al. (44) recently found that the binding occurred even after enzymatic removal of heparan sulfate and could not be competed by $100-$ fold molar excess of FGF2. In contrast with this study, it has been recently reported that the heparan sulfate chains of glypicans act as low-affinity receptors for endostatin (45). Thus, endostatin appears to have a complex repertoire of in vivo interactions, as further exemplified by the cell-free binding assays in which several basement membrane molecules, including fibulin, nidogen, and perlecan, bind to endostatin with variable affinities (43).

During homeostasis, endostatin could be immobilized by these potentially complex interactions and could be liberated during cancer growth by overproduced proteolytic enzymes. If heparan sulfate chains are indeed protective, an interesting possibility would be that heparanase could trigger the initial activation of endostatin and related molecules. Since endostatin and perlecan are associated with the elastic lamellae of aorta and other elastic vessels, and since elastase can discharge HSPGs, it is possible that pro- and antiangiogenic stimuli can be released during remodeling and tumor invasion. Future analyses of these interactions will become instrumental in understanding the endogenous control of angiogenesis inhibitors.

\section{Conclusions and clinical implications}

Four major classes of HSPGs have been shown to play potent regulatory roles in angiogenesis. A typical feature is a dual, positive and negative, activity. This dichotomy is further stressed by a differential binding of domains within the heparan sulfate chains for FGF or VEGF ligands and their tyrosine kinase receptors. Specificity is translated into action by the remarkable diversity of heparan sulfate chains and by the various modules of HSPG protein cores. Because of the information density in heparan sulfate chains, which can greatly exceed that of nucleic acids, the chemical diversity of heparan 
sulfate, coupled with the richness of protein cores, can provide a highly sensitive system for cells to generate unique responses to angiogenic factors.

When a quiescent capillary is stimulated to undergo angiogenesis, the angiogenic stimuli often enhance the synthesis and deposition of perlecan adjacent to endothelial cell surfaces and intensify its cell surface accessibility (Figure 2, top). Perlecan, thus positioned, would act as a coreceptor to deliver growth factors such as FGFs or VEGFs from heparan sulfate- and protein core-binding sites to their high-affinity cell surface receptors, thereby stimulating mitosis (Figure 2, middle). Other full-time HSPGs, such as syndecans and glypicans, are also directly involved in regulating the development of new blood vessels. Molecules that potentiate the activity of FGFs, such as FGF-BP, could be concurrently released in an active form from processing of the perlecan protein core. After the ensuing burst of endothelial cell proliferation, migration, and capillary morphogenesis has subsided, localized release of proteases, such as cathepsin L or MMPs, would degrade perlecan, thereby removing the angiogenic stimulus, and act on collagen XVIII to liberate endostatin. This would in turn maintain endothelial cells in a quiescent, differentiated state (Figure 2, bottom). Alteration of such equilibria between positive and negative signals is a hallmark of pervasive diseases such as cancer and atherosclerosis.

As human lifespan increases, angiogenic cues and their regulators will undoubtedly play a more prominent role in several disease states in which an abnormal vascular proliferation is the norm. Obvious clinical implications of the data reviewed above include gene therapies and pharmacological approaches directed at either hindering proangiogenic stimuli, such as perlecan, or augmenting the production of endogenous antiangiogenic factors, such as endostatin. Identification of endogenous precursors of angiogenesis inhibitors and their subsequently processed isoforms in the context of tumor progression would aid in elucidating the key steps involved in the regulation of the newly formed blood supply. This would in turn help in defining a tailored antiangiogenic therapy for specific tumors. For instance, targeting perlecan could be beneficial in the treatment of certain highly vascularized tumors that are not responding to classical therapies. Circulating endostatin or endostatin-like molecules could be beneficial in retarding tumor growth by slowing down the formation of a new blood supply. This would also allow cooperative action of cytostatic or chemotherapeutic drugs. Specific antiangiogenic therapies could be tailored for the individual patient once more knowledge is accumulated regarding the genetic and biological behavior of specific forms of cancer.

\section{Acknowledgments}

This work was supported by grants CA-39481, CA47282, and HL-53590 from the NIH and grant DAMD17-00-1-0663 from the Department of the Army. We thank J. Sweeney for artistic work and C.C. Clark for critical reading of the manuscript. We wish to extend a sincere apology to our colleagues whose work was not cited because of space limitations. Interested readers can find additional references in the suggested reading list at [www.jci.org/cgi/content/full/108/03/349/DC1].

1. Erickson, A.C., and Couchman, J.R. 2000. Still more complexity in mammalian basement membranes. J. Histochem. Cytochem. 48:1291-1306.

2. Lander, A.D., and Selleck, S.B. 2000. The elusive functions of proteoglycans: in vivo veritas. J. Cell Biol. 148:227-232.

3. Bernfield, M., et al. 1999. Functions of cell surface heparan sulfate proteoglycans. Annu. Rev. Biochem. 68:729-777.

4. Iozzo, R.V. 1998. Matrix proteoglycans: from molecular design to cellular function. Annu. Rev. Biochem. 67:609-652.

5. Filmus, J., and Song, H.H. 2000. Glypicans. In Proteoglycans: structure, biology and molecular interactions. R.V. Iozzo, editor. Marcel Dekker Inc. New York, New York, USA. 161-176.

6. Neumann, F.R., et al. 2001. An alternative amino-terminus expressed in the central nervous system converts agrin to a type II transmembrane protein. Mol. Cell. Neurosci. 17:208-225.

7. Dunlevy, J.R., and Hassell, J.R. 2000. Heparan sulfate proteoglycans in basement membranes: perlecan, agrin and collagen XVIII. In Proteoglycans: structure, biology and molecular interactions. R.V. Iozzo, editor. Marcel Dekker Inc. New York, New York, USA. 275-326.

8. Zatterstrom, U.K., Felbor, U., Fukai, N., and Olsen, B.R. 2000. Collagen $\mathrm{XVIII/endostatin} \mathrm{structure} \mathrm{and} \mathrm{functional} \mathrm{role} \mathrm{in} \mathrm{angiogenesis.} \mathrm{Cell}$ Struct. Funct. 25:97-101.

9. Li, D., Clark, C.C., and Myers, J.C. 2000. Basement membrane zone type $\mathrm{XV}$ collagen is a disulfide-bonded chondroitin sulfate proteoglycan in human tissues and cultured cells. J. Biol. Chem. 275:22339-22347.

10. Perrimon, N., and Bernfield, M. 2000. Specificities of heparan sulphate proteoglycans in developmental processes. Nature. 404:725-728.

11. Baeg, G.-H., Lin, X., Khare, N., Baumgartner, S., and Perrimon, N. 2001. Heparan sulfate proteoglycans are critical for the organization of the extracellular distribution of Wingless. Development. 128:87-94.

12. Aviezer, D., et al. 1994. Perlecan, basal lamina proteoglycan, promotes basic fibroblast growth factor-receptor binding, mitogenesis, and angiogenesis. Cell. 79:1005-1013.

13. Bonneh-Barkay, D., et al. 1997. Identification of glypican as a dual modulator of the biological activity of fibroblast growth factor. J. Biol. Chem. 272:12415-12421.

14. Clasper, S., et al. 1999. Inducible expression of the cell surface heparan sulfate proteoglycan syndecan-2 (fibroglycan) on human activated macrophages can regulate fibroblast growth factor action. J. Biol. Chem. 274:24113-24123.

15. Jones, M., Tussey, L., Athanasou, N., and Jackson, D.G. 2000. Heparan sulfate proteoglycan isoforms of the CD44 hyaluronan receptor induced in human inflammatory macrophages can function as paracrine regulators of fibroblast growth factor action. J. Biol. Chem. 275:7964-7974.

16. Nugent, M.A., and Iozzo, R.V. 2000. Fibroblast growth factor-2. Int. J. Biochem. Cell Biol. 32:115-120.

17. Fannon, M., Forsten, K.E., and Nugent, M.A. 2000. Potentiation and inhibition of bFGF binding by heparin: a model for regulation of cellular response. Biochemistry. 39:1434-1445.

18. Padera, R., Venkataraman, G., Berry, D., Godavarti, R., and Sasisekharan, R. 1999. FGF-2/fibroblast growth factor receptor/heparin-like glycosaminoglycan interactions: a compensation model for FGF-2 signaling. FASEB J. 13:1677-1687.

19. Gengrinovitch, S., et al. 1999. Glypican-1 is a VEGF VES $_{165}$ binding proteoglycan that acts as an extracellular chaperone for $\mathrm{VEGF}_{165}$. J. Biol. Chem. 274:10816-10822.

20. Iozzo, R.V., Cohen, I.R., Grässel, S., and Murdoch, A.D. 1994. The biology of perlecan: the multifaceted heparan sulphate proteoglycan of basement membranes and pericellular matrices. Biochem. J. 302:625-639.

21. Handler, M., Yurchenco, P.D., and Iozzo, R.V. 1997. Developmental expression of perlecan during murine embryogenesis. Dev. Dyn. 210:130-145.

22. Aviezer, D., Iozzo, R.V., Noonan, D.M., and Yayon, A. 1997. Suppression of autocrine and paracrine functions of basic fibroblast growth factor by stable expression of perlecan antisense cDNA. Mol. Cell. Biol. 17:1938-1946.

23. Adatia, R., et al. 1998. Suppression of invasive behavior of melanoma cells by stable expression of anti-sense perlecan cDNA. Ann. Oncol. 8:1257-1261.

24. Rapraeger, A.C., Guimond, S., Krufka, A., and Olwin, B.B. 1994. Regulation by heparan sulfate in fibroblast growth factor signaling. Methods Enzymol. 245:219-240.

25. Sharma, B., et al. 1998. Antisense targeting of perlecan blocks tumor growth and angiogenesis in vivo. J. Clin. Invest. 102:1599-1608.

26. Mongiat, M., et al. 2000. The protein core of the proteoglycan perlecan 
binds specifically to fibroblast growth factor-7. J. Biol. Chem. 275:7095-7100.

27. Göhring, W., Sasaki, T., Heldin, C.H., and Timpl, R. 1998. Mapping of the binding of platelet-derived growth factor to distinct domains of the basement membrane proteins BM-40 and perlecan and distinction from the BM-40 collagen-binding epitope. Eur. J. Biochem. 255:60-66.

28. Mongiat, M., et al. 2001. Fibroblast growth factor-binding protein is a novel partner for perlecan protein core. J. Biol. Chem. 276:10263-10271.

29. Wu, D., Kan, M., Sato, G.H., Okamoto, T., and Sato, J.D. 1991. Characterization and molecular cloning of a putative binding protein for heparin-binding growth factors. J. Biol. Chem. 266:16778-16785.

30. Czubayko, F., et al. 1997. A secreted FGF-binding protein can serve as the angiogenic switch in human cancer. Nat. Med. 3:1137-1140.

31. Mathiak, M., Yenisey, C., Grant, D.S., Sharma, B., and Iozzo, R.V. 1997. A role for perlecan in the suppression of growth and invasion in fibrosarcoma cells. Cancer Res. 57:2130-2136.

32. Marchisone, C., et al. 2000. Phenotypic alterations in Kaposi's sarcoma cells by antisense reduction of perlecan. Pathol. Oncol. Res. 6:10-17.

33. Sharma, B., and Iozzo, R.V. 1998. Transcriptional silencing of perlecan gene expression by interferon- $\gamma$. J. Biol. Chem. 273:4642-4646.

34. Pellegrini, L., Burke, D.F., von Delft, F., Mulloy, B., and Blundell, T.L. 2000. Crystal structure of fibroblast growth factor receptor ectodomain bound to ligand and heparin. Nature. 407:1029-1034.

35. Yu, W.-H., and Woessner, J.F., Jr. 2000. Heparan sulfate proteoglycans as extracellular docking molecules for matrilysin (matrix metalloproteinase 7). J. Biol. Chem. 275:4183-4191.

36. Whitelock, J.M., Murdoch, A.D., Iozzo, R.V., and Underwood, P.A. 1996.
The degradation of human endothelial cell-derived perlecan and release of bound basic fibroblast growth factor by stromelysin, collagenase, plasmin and heparanases. J. Biol. Chem. 271:10079-10086.

37. O'Reilly, M.S., et al. 1997. Endostatin: an endogenous inhibitor of angiogenesis and tumor growth. Cell. 88:277-285.

38. Musso, O., et al. 2001. Tumor progression is associated with a significant decrease in the expression of the endostatin precursor collagen XVIII in human hepatocellular carcinomas. Cancer Res. 61:45-49.

39. Yamaguchi, N., et al. 1999. Endostatin inhibits VEGF-induced endothelial cell migration and tumor growth independently of zinc binding. EMBO J. 18:4414-4423.

40. Sasaki, T., et al. 1999. Structural basis and potential role of heparin/heparan sulfate binding to the angiogenesis inhibitor endostatin. EMBO J. 18:6240-6248.

41. Rehn, M., et al. 2001. Interaction of endostatin with integrins implicated in angiogenesis. Proc. Natl. Acad. Sci. USA. 98:1024-1029.

42. Kim, Y.-M., et al. 2000. Endostatin inhibits endothelial and tumor cellular invasion by blocking the activation and catalytic activity of matrix metalloproteinase 2. Cancer Res. 60:5410-5413.

43. Sasaki, T., et al. 2000. Endostatins derived from collagens XV and XVIII differ in structural and binding properties, tissue distribution and antiangiogenic activity. J. Mol. Biol. 301:1179-1190.

44. Chang, Z., Choon, A., and Friedl, A. 2000. Endostatin binds to blood vessels in situ independent of heparan sulfate and does not compete for fibroblast growth factor-2 binding. Am. J. Pathol. 155:71-76.

45. Karumanchi, S.A., et al. 2001. Cell surface glypicans are low-affinity endostatin receptors. Mol.Cell. 7:811-822. 